\title{
T-wave Morphology Restitution Dependency with Heart Rate Range and Its Association with Sudden Cardiac Death in Chronic Heart Failure
}

\author{
Julia Ramírez ${ }^{1}$, Michele Orini ${ }^{2}$, Esther Pueyo ${ }^{1}$ Pablo Laguna ${ }^{1}$ \\ ${ }^{1}$ Biomedical Signal Interpretation and Computational Simulation (BSICoS) group, Aragón Institute \\ of Engineering Research (I3A), IIS Aragón, University of Zaragoza, Zaragoza, Spain, and \\ Biomedical Research Networking Center in Bioengineering, Biomaterials and Nanomedicine \\ (CIBER-BBN), Zaragoza, Spain \\ ${ }^{2}$ Institute of Cardiovascular Science, University College London, London, UK
}

\begin{abstract}
The index of T-wave morphology restitution (TMR) has recently been presented as a sudden cardiac death (SCD) predictor in a population of chronic heart failure (CHF) patients. This index quantifies the level of variation in the T-wave morphology per RR interval (RRI) increment. The impact of using different definitions of ranges of RRI $(\triangle R R I)$ and the study of the interaction between TMR and $\triangle R R I$ are relevant for both the optimization and the mechanistic interpretation of the index. This study aims to investigate the interaction between $\triangle R R I$ and TMR and to assess whether different definitions of $\Delta R R I$ (patientsindependent or patient-specific) may affect the predictive value of TMR. Holter ECG recordings from 651 patients with $C H F$, including SCD, victims of other causes and survivors, were analyzed. Mann-Whitney test was used to evaluate significant differences between SCD victims and the rest of patients. In the patient-independent analysis, TMR values only showed significant differences when $0.25 s \leq \Delta R R \leq 0.3 \mathrm{~s}$, while in the patient-specific analysis $T M R$ values were significantly different when $\Delta R R \geq 0.6$ of the maximum $R R$ range, $\Delta R R_{\max }$. In conclusion, the index TMR is a predictor of SCD robust to variations in $\triangle R R$ values evaluated in segments comprising at least 0.6 of $\Delta R R_{\max }$.
\end{abstract}

\section{Introduction}

The action potential duration (APD) restitution (APDR) curve represents the interaction between local APD and local cycle length [1]. The morphology of the T-wave reflects the distribution of the repolarization sequence along the ventricle [2-4]. Therefore, the spatio-temporal inhomogeneity of the ventricular repolarization process as a response to changes in heart rate may be captured by an index measuring T-wave morphological changes.
In a recent study, the index of T-wave morphology restitution (TMR) was proposed and proved to predict sudden cardiac death (SCD) in a population of 651 chronic heart failure (CHF) patients [5]. The TMR is calculated as the difference in the morphology of two T-waves measured at two RR intervals (RRI) defining the maximum intrasubject RRI range, $\Delta \mathrm{RRI}$, and normalized by $\Delta \mathrm{RRI}$. The impact of using different definitions of $\Delta R R I$ and the study of the interaction between TMR and $\Delta R R I$ are relevant for both the optimization and the mechanistic interpretation of the index. This study aims to investigate the interaction between $\triangle \mathrm{RRI}$ and TMR and to assess whether different definitions of $\Delta R R I$ may affect the predictive value of TMR.

In this study we calculated the evolution of the index TMR with an patient-independent and patient-specific change in $\triangle R R$. Then, we calculated the SCD predictive value of TMR at each value of $\Delta R R$ and we compared the patient-independent and patient-specific results.

\section{Materials and Methods}

\subsection{Materials}

The study population included 651 consecutive patients with symptomatic CHF corresponding to NYHA classes II and III enrolled in the MUSIC study, a prospective, multicenter study designed to assess risk predictors for cardiovascular mortality in ambulatory patients with $\mathrm{CHF}$ [6]. A two- or three-lead 24-hour Holter ECG sampled at $200 \mathrm{~Hz}$ was recorded in each patient at enrolment using ELA Medical equipment (Sorin Group, Paris, France). Baseline demographic and clinical data in sinus rhythm were available for the analysis. The MUSIC study included patients with both reduced and preserved LVEF, ranging from $10 \%$ to $70 \%$. The study protocol was approved by the institutional investigation committees and all patients signed informed consent. No medications were withdrawn during Holter 

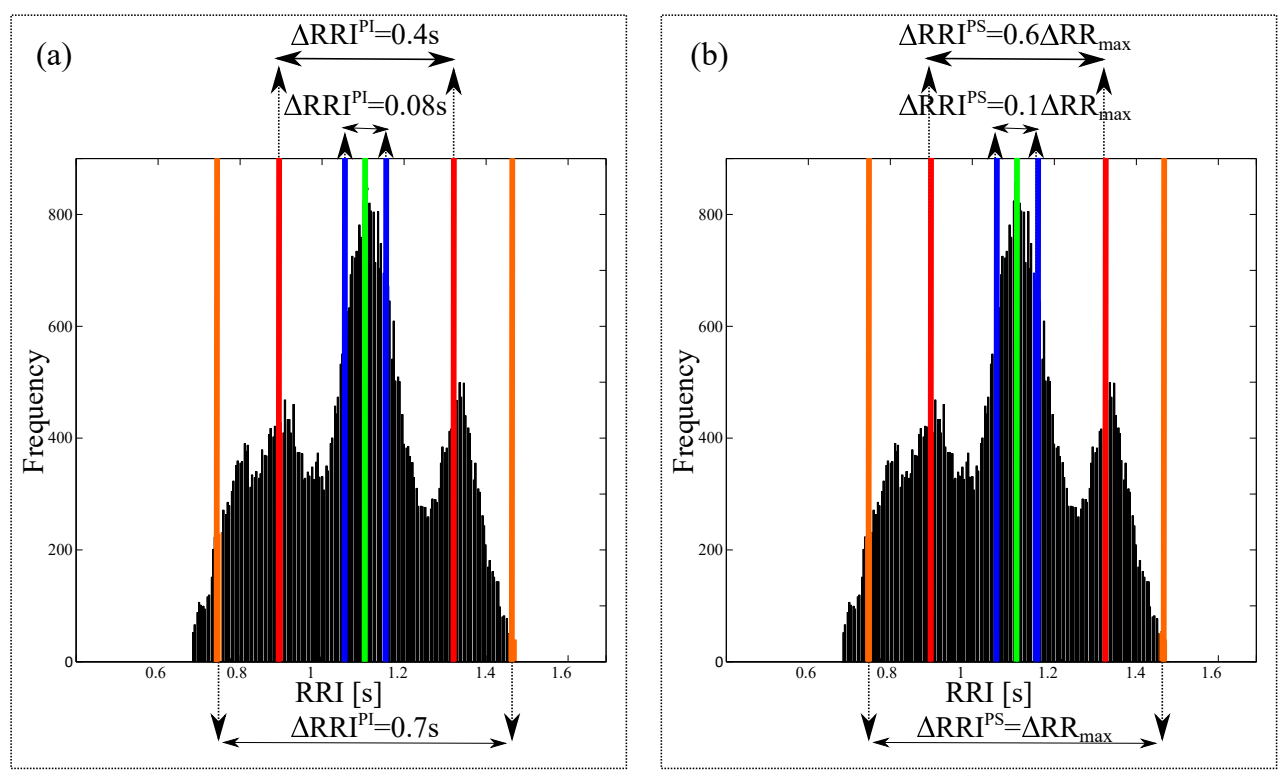

Figure 1. Selection of patient-independent (a) and patient-specific (b) ranges. Histogram of RR divided into 10-ms wide bins. Pairs of bins are selected by using fixed $\Delta$ RRI (a) or $\Delta$ RRI patient-specific to the maximum intra-subject $\Delta R R$.

monitoring.

Patients were followed up every 6 months for a median of 48 months. SCD was defined as (1) a witnessed death occurring within 60 minutes from the onset of new symptoms unless a cause other than cardiac failure was obvious, (2) an unwitnessed death $(<24$ hours) in the absence of preexisting progressive circulatory failure or other causes of death, or (3) death during attempted resuscitation. End points were reviewed and classified by the MUSIC Study Endpoint Committee.

\subsection{ECG Pre-processing}

Preprocessing of the ECG signals was performed using custom-written software and included low pass filtering at $40 \mathrm{~Hz}$ to remove electric and muscle noise, cubic splines interpolation for baseline wander removal, and ectopic beats detection.

Principal Component Analysis (PCA) was applied over the two-or-three available ECG leads to emphasize the energy of the T-wave and improve its delineation [7]. The PCA training matrix was built by only considering the samples from the T-waves on each lead. First, a singlelead-and-rules delineation technique was applied to select the samples from the T-wave and compute the PCA training matrix. Then, the first principal component was computed and delineated using a single-lead technique [8]. From the delineation marks, the RR interval series was obtained and the T-waves were selected using the known delimitation marks.

\subsection{T-wave Morphology Restitution Using Patient-independent RR Ranges}

Automatic quantification of the T-wave morphology restitution for patient-independent $R R$ ranges was performed on every ECG recording in 4 steps:

1. Selection of patient-independent $\Delta$ RRIs: First, the histogram of the RR series was calculated during the entire 24-h recording, and it was divided into bins of $10 \mathrm{~ms}$ wide, showing a minimum frequency equal to 50 . Next, a series of $\Delta$ RRI values, $\Delta R R I^{\mathrm{PI}}(i)$, was defined as:

$$
\begin{aligned}
\Delta R R I^{\mathrm{PI}}(i) & =R R I_{2}^{\mathrm{pl}}(i)-R R I_{1}^{\mathrm{pl}}(i), \quad i=1, \cdots, N \\
R R I_{1}^{\mathrm{P1}}(i) & =R R I_{m}-10 i \\
R R I_{2}^{\mathrm{P1}}(i) & =R R I_{m}+10 i
\end{aligned}
$$

where $R R I_{m}$ is the median RRI in the histogram and $N$ is the maximum number of symmetric bins around $R R I_{m}$ (Fig. 1 (a)). Then, the T-waves corresponding to the beats associated with $R R I_{1}^{\mathrm{pI}}(i)$ and $R R I_{2}^{\mathrm{PI}}(i)$ were considered for the analysis.

2. Mean warped T-waves: The mean warped T-waves of the T-waves within $R R I_{1}^{\mathrm{PI}}(i)$ and $R R I_{2}^{\mathrm{PI}}(i)$ were calculated by time-warping as in [9]. Each mean warped T-waves is a representative of the average $\mathrm{T}$-wave morphology at $R R I_{1}^{\mathrm{pl}}(i)$ and $R R I_{2}^{\mathrm{PI}}(i)$, and compensate for the possible morphological differences introduced by eventual different histories of RRIs [5].

3. Quantification of the T-wave morphological differences: The morphological differences between the $i$-th 
(a)
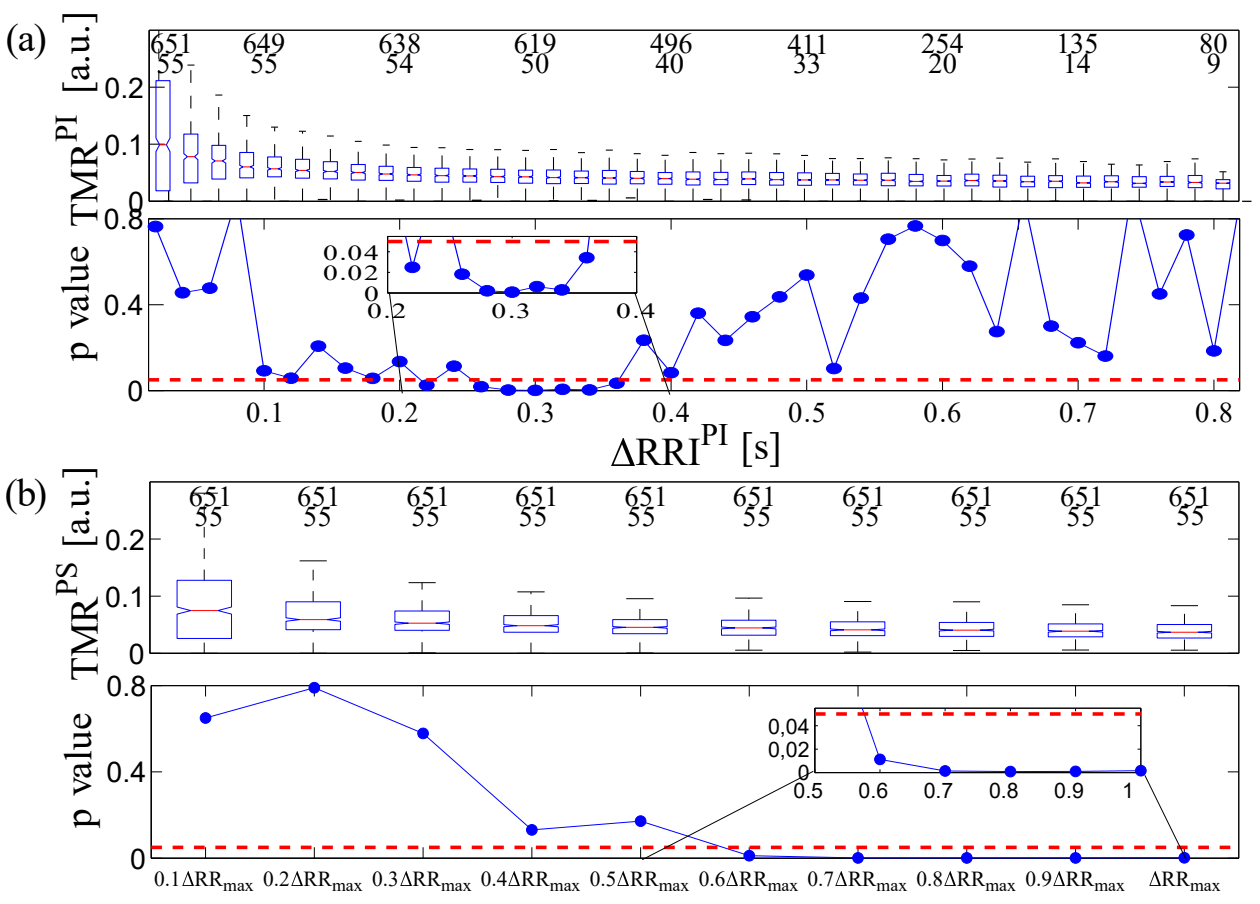

$\Delta \mathrm{RRI}^{\mathrm{PS}}[\mathrm{s}]$

Figure 2. T-wave morphology restitution series using patient-independent (a) and patient-specific (b) RR ranges. Top panels show the boxplots of the TMR values, calculated across subjects, with respect to the patient-independent (a) and patient-specific (b) RR ranges. Bottom panels show the p-values of the Mann-Whitney test when comparing TMR values in SCD victims with respect to the rest of patients. The red dashed horizontal line marks the significance level, i.e. $\mathrm{p}=0.05$. A zoom of the significant p-values is also provided. The first and second raw of numbers on top of each boxplot describes the total number of patients and the total number of SCD victims, respectively, in each boxplot.

pair of mean warped T-waves, corresponding to $R R I_{1}^{\mathrm{PI}}(i)$ and $R R I_{2}^{\mathrm{Pl}}(i)$, were quantified by $d_{w}^{\mathrm{Pl}}(i)$, a time-warpingbased index measuring the level of variation in the T-wave temporal domain [9]. Before applying the methodology, the gravity centres of both mean warped T-waves were aligned to only account on changes in the T-wave morphology, and not on global shifts (see [9] for details).

4. T-wave morphology restitution series: The series $T M R^{\mathrm{PI}}(i)$ was, then, calculated by normalizing the variations in the temporal domain of each pair of mean warped $\mathrm{T}$-waves by the corresponding RR range change:

$$
T M R^{\mathrm{PI}}(i)=\frac{d_{w}^{\mathrm{P}}(i)}{\Delta R R I^{\mathrm{PI}}(i)}
$$

\subsection{T-wave Morphology Restitution Using Patient-specific RR Ranges}

The range of RR is highly subject-dependent and, thus, imposing a fixed increment in the range of RR for the TMR calculation might be a limitation. Then, for each subject, the maximum intra-subject RRI range, $\Delta R R_{\max }$, was divided into ten equally-spaced bins as follows (Fig. 1 (b)):

$$
\begin{aligned}
\Delta R R I^{\mathrm{PS}}(j) & =0.1 j \Delta R R_{\max }, \quad j=1, \cdots, 10 \\
R R I_{1}^{\mathrm{ps}}(j) & =R R I_{m}-\frac{0.1 j \Delta R R_{\max }}{2}, \\
R R I_{2}^{\mathrm{ps}}(j) & =R R I_{m}+\frac{0.1 j \Delta R R_{\max }}{2},
\end{aligned}
$$

and the $\mathrm{T}$-waves corresponding to the RR intervals within $R R I_{1}^{\mathrm{PS}}(j)$ and $R R I_{2}^{\mathrm{PS}}(j)$ were considered for the subsequent analysis. The calculation of the mean warped Twaves and the computation of the series of temporal variations, $d_{w}^{\mathrm{ps}}(i)$, was performed as described in the previous section. Finally, the series $T M R^{\mathrm{ps}}(i)$ was obtained as:

$$
T M R^{\mathrm{ps}}(j)=\frac{d_{w}^{\mathrm{ps}}(j)}{\Delta R R I^{\mathrm{Ps}}(j)} .
$$

\subsection{Statistical Analysis}

Two-tailed Mann-Whitney test was used for univariate comparison of quantitative data. 


\section{Results and Discussion}

Top panels in Fig. 2 show the boxplot of TMR, calculated across subjects, with respect to the patientindependent (a) and patient-specific (b) values of $\Delta R R$. The first and second raw of numbers on top of each boxplot describes the total number of patients and the total number of SCD victims, respectively. In (a), this number is shown for each boxplot out of three due to the large number of boxplots. The p-value of Mann-Whitney's U test for comparison between SCD victims and the rest of patients is shown in the bottom panels, where each dot corresponds to a boxplot. The red dashed horizontal line marks the significance level, i.e. $\mathrm{p}=0.05$.

$T M R^{\text {PI }}$ was significantly different in SCD victims as compared to the rest of patients for $\Delta R R I$ values between 0.25 and $0.3 \mathrm{~s}$ (a). Moreover, only half the total number of patients had $\Delta \mathrm{RR}$ values above $0.5 \mathrm{~s}$. This may be due to the fact that $\Delta R R I^{\mathrm{PI}}(i)$ was set to be symmetrical around $R R I_{m}$ and the histogram could be slightly skewed (Fig. 1 (a)). $T M R^{\mathrm{PS}}$ median values were consistently significantly different in SCD victims than in the rest of patients from $\Delta R R I^{\mathrm{PS}}(j) \geq 0.6 \Delta \mathrm{RR}_{\max }$. In addition, the number of patients in each boxplot was the same since the different values of $\Delta R R I^{\mathrm{PS}}(j)$ are proportional to $\Delta \mathrm{RR}_{\max }$.

Also, it can be appreciated that both the median value and variability of $T M R^{\mathrm{PI}}$ and $T M R^{\mathrm{PS}}$ are slightly larger for lower $\Delta R R I^{\mathrm{PI}}(j)$ and $\Delta R R I^{\mathrm{PS}}(j)$. This suggests that the morphological changes in the T-wave morphology as a response to small increments in $\Delta R R I^{\mathrm{PI}}(j)$ and $\Delta R R I^{\mathrm{PS}}(j)$ are comparable to those produced by noise.

$T M R^{\mathrm{PI}}$ and $T M R^{\mathrm{PS}}$ values showed the lowest $\mathrm{p}$ value for SCD risk stratification when calculated at $\Delta R R I^{\mathrm{PI}}(j)=0.3 \mathrm{~s}(60000 /(1000-150)-60000 /(1000+150)=18$ beats per minute) $(\mathrm{p}=0.0008)$ (Figure $2(\mathrm{a}))$, and when calculated at $\Delta R R I^{\mathrm{PS}}(j)=0.8 \Delta \mathrm{RR}_{\max }(\mathrm{p}=0.0008)$ (Figure 2 (b)), respectively.

\section{Conclusions}

The index of T-wave morphology restitution (TMR), hypothesized to be related to variations in dispersion of repolarization due to changes in heart rate, is a robust predictor of SCD when considering values of RR range as a fraction $(>0.6)$ of the maximum intra-subject $R R$ range or a fixed RR range between 0.25 and $0.35 \mathrm{~s}$. Our results show that SCD victims manifest a significantly higher variation in the T-wave morphology per RR increment than the rest of patients when the RR range exceeds 0.6 of their maximum intra-subject RR range. When calculating TMR using patient-independent values of $\mathrm{RR}$ range, the predictive value of this index was not consistently significant as the $\mathrm{RR}$ range increases. Our results suggest that the variation in the T-wave morphology is patient-specific to the intra- subject range and, thus, patient-specific RR ranges should be considered in future studies using TMR for SCD risk prediction.

\section{Acknowledgements}

This work was supported in part by projects TIN2013-41998-R and DPI2016-75458-R from Spanish Ministry of Economy and Competitiveness (MINECO), Spain, and by Aragón Government, Spain and from European Social Fund (EU) through BSICoS group. The computation was performed at the High Performance computing platform of the NANBIOSIS ICTS, CIBER-BBN and I3A, Zaragoza, Spain.

\section{References}

[1] Orini M., Taggart P., Srinivasan N., Hayward M. and Lambiase P.D., "Interactions between activation and repolarization restitution properties in the intact human heart: In vivo whole-heart data and mathematical description," Plos One, vol. 11, no. 9, p. e0161765, 2016.

[2] Burgess M. J., "Relation of ventricular repolarization to electrocardiographic T wave-form and arrhythmia vulnerability," Am J Physiol, vol. 236, no. 3, pp. H391-H402, 1979.

[3] Shimizu W. and Antzelevitch C., "Cellular basis for long QT, transmural dispersion of repolarization, and torsade de pointes in the long QT syndrome," J Electrocardiol, vol. 32, no. Suppl, pp. 177-184, 1999.

[4] Gima K. and Rudy Y., "Ionic current basis of electrocardiographic waveforms: a model study," Circ Res, vol. 90, no. 8, pp. 889-896, 2002.

[5] Ramírez J., Orini M., Mincholé A., Monasterio V., Cygankiewicz I., De Luna A. B., et. al., "T-wave morphology restitution predicts sudden cardiac death in patients with chronic heart failure," J Am Heart Assoc, vol. In Press, 2017.

[6] Vázquez R., Bayés-Genís A., Cygankiewicz I., Pascual-Figal D., Grigorian-Shamagian L., Pavon R., et. al., "The MUSIC Risk score: a simple method for predicting mortality in ambulatory patients with chronic heart failure.," European Heart Journal, vol. 30, pp. 1088-1096, 2009.

[7] Ramírez J., Mincholé A., Laguna P. and Pueyo E., "Characterization of cardiac repolarization response to heart rate changes provoked by a Tilt test," in Computing in Cardiology, vol. 39, pp. 673-676, 2012.

[8] Martínez J.P., Almeida R., Olmos S., Rocha A.P. and Laguna P., "A wavelet-based ECG delineator: evaluation on standard databases," IEEE Transactions on Biomedical Engineering, vol. 51, pp. 570-581, 2004.

[9] Ramírez J., Orini M., Tucker J.D., Pueyo E. and Laguna P., "Variability of ventricular repolarization dispersion quantified by time-warping the morphology of the T-waves.," IEEE Trans Biomed Eng, vol. DOI:10.1109/TBME.2016.2614899, 2016.

Address for correspondence:

Julia Ramírez, Campus Río Ebro, I+D Building, D-5.01.1B, c $\backslash$ Mariano Esquillor, s/n, 50018 Zaragoza (Spain),

Julia.Ramirez@unizar.es 